\title{
Multicenter prospective study on the histological diagnosis of gastric cancer by narrow band imaging-magnified endoscopy with and without acetic acid
}

\section{다)(1) $(9)$}

\section{Authors}

Takaaki Kishino ${ }^{1,2}$, Tsuneo Oyama1, Keita Funakawa ${ }^{3}$, Eiji Ishii ${ }^{4}$, Tetsuro Yamazato ${ }^{5}$, Kotaro Shibagaki ${ }^{6}$, Tadashi Miike $^{7}$, Tokuma Tanuma ${ }^{8}$, Yasuharu Kuwayama ${ }^{9}$, Manabu Takeuchi ${ }^{10}$, Yoko Kitamura ${ }^{2}$

Institutions

1 Department of Endoscopy, Saku Central Hospital Advanced Care Center, Saku, Japan

2 Department of Gastroenterology, Nara City Hospital, Higashikidera-cho, Nara, Japan

3 Department of Gastroenterology, Kagoshima University School of Medical and Dental Sciences, Kagoshima, Japan

4 Department of Gastroenterology, Kameda Medical Center, Kamogawa, Japan

5 Department of Gastroenterology, Tokyo Metropolitan Cancer Detection Center, Fuchu, Japan

6 Department of Gastroenterology, Tottori Municipal Hospital, Tottori, Japan

7 Department of Gastroenterology, University of Miyazaki, Miyazaki, Japan

8 Department of Gastroenterology, Teine Keijinkai Hospital, Sapporo, Japan

9 Department of Gastroenterology, Tokushima Red Cross Hospital, Komatsushima, Japan

10 Department of Gastroenterology, Niigata University Medical and Dental Hospital, Niigata, Japan

submitted 11.7.2018

accepted after revision $\quad 8.10 .2018$

Bibliography

DOI https://doi.org/10.1055/a-0806-7275 |

Endoscopy International Open 2019; 07: E155-E163

(c) Georg Thieme Verlag KG Stuttgart · New York

ISSN 2364-3722

Corresponding author

Takaaki Kishino, MD, Department of Gastroenterology, Nara City Hospital, 1-50-1 Higashikideracho, Nara 6308305, Japan

Fax: $+81-742222478$

t-kishino@nara-jadecom.jp
ABSTRACT

Background and study aims The usefulness of endoscopy for diagnosing histological type remains unclear. This study aimed to examine the diagnostic accuracy of white light endoscopy (WLE), magnified endoscopy with narrow band imaging (NBI-ME), and NBI-ME with acetic acid enhancement (NBI-AA) for histological type of gastric cancer. Patients and methods Patients with depressed-type gastric cancers resected by endoscopic submucosal dissection were prospectively enrolled, and 221 cases were analyzed. Histological type was diagnosed by WLE, followed by NBIME and NBI-AA. Histological type was classified into differentiated adenocarcinoma and undifferentiated adenocarcinoma. Histological type was diagnosed based on lesion color in WLE, surface patterns (pit, villi, and unclear) and vascular irregularities in NBI-ME, and surface patterns in NBI-AA.

Results Histological types of target areas were differentiated adenocarcinoma and undifferentiated adenocarcinoma in 206 and 15 cases, respectively. Diagnostic accuracy of WLE, NBI-ME, and NBI-AA for the histological type was 96.4\% (213/221), 96.8\% (214/221), and 95.5\% (211/221), respectively. No significant differences were observed among modalities. Positive predictive value based on endoscopic findings in NBI-ME was $98.0 \%$ (149/152) for the villi pattern, $100 \%$ (19/19) for the irregular pit pattern, $100 \%$ (9/9) for the unclear surface pattern with a vascular network, $90.3 \%$ (28/31) for the unclear surface pattern with mild vascular irregularity, and $88.9 \%(8 / 9)$ for the unclear surface pattern with severe vascular irregularity.

Conclusions NBI-ME and NBI-AA did not show any advantages over WLE for diagnostic accuracy. Villi pattern, irregular pit pattern, and vascular network may be useful for identifying differentiated adenocarcinoma. 


\section{Introduction}

Marked advances in endoscopic treatment for early gastric cancer have been achieved by endoscopic submucosal dissection (ESD) [1]. In the Japanese guidelines for ESD and endoscopic mucosal resection for early gastric cancer [2], the Japan Gastroenterological Endoscopy Society reported that endoscopic resection is recommended for tumors with a very low potential for lymph node metastasis and suitable for en bloc resection. Histological type is a risk factor for lymph node metastasis [3, 4] and affects patient prognosis [5]. Indications for endoscopic resection differ between differentiated and undifferentiated carcinomas because the latter have a greater potential for lymph node metastasis than the former $[1,3,4]$. Therefore, histological diagnosis before treatment selection is important for deciding a suitable treatment plan [6].

Histological diagnosis is achieved before endoscopic resection based on biopsies, but does not always reflect the main histological type [7]; the biopsy site only reflects a small part of the lesion. Therefore, difficulties are associated with diagnosing the histological type of mixed histological-type gastric cancer based on biopsies. On the other hand, endoscopy has a greater capacity than biopsies to diagnose histological type because it is possible to observe the whole lesion. A magnified endoscopy and narrow band image (NBI-ME) system has recently been developed and enables surface and vascular structures to be evaluated in detail. The usefulness of NBI-ME for diagnosing lateral extension and differentiation of neoplastic and non-neoplastic lesions has been demonstrated [8-11]. Moreover, the usefulness of NBI-ME in histological diagnosis of gastric cancer has been reported [12-14]. Nakayoshi et al. found using NBI-ME that a fine network vascular pattern was present in differentiated adenocarcinoma, while a corkscrew vascular pattern was observed in undifferentiated adenocarcinoma [13].

Kadowaki et al. reported that NBI-ME with acetic acid (NBIAA) was useful for evaluating the surface structure of gastric cancer [15]. Yagi et al. showed that this modality enabled clear observation of crypts in the glandular epithelium as a result of reversible alterations in the molecular structures of cellular proteins [16]. However, a multicenter prospective study has not yet been conducted on the diagnostic accuracy of histological type by endoscopy. Therefore, we herein performed a multicenter prospective study to evaluate the usefulness of NBI-ME and NBI-AA for diagnosing histological type of gastric cancer. In a secondary evaluation, we investigated diagnostic yields of $\mathrm{NBI}-\mathrm{ME}$ and NBI-AA based on endoscopic findings.

\section{Patients and methods}

\section{Study design}

The current study was conducted as a multicenter prospective trial at three tertiary care academic centers and seven community-based hospitals in Japan. The study protocol was approved by the Institutional Review Boards at all institutions. This trial was also registered with the UMIN Clinical Trials Registry (clinical trial registration number: UMIN 000006042).

\section{Patients}

We prospectively recruited 250 consecutive patients who were candidates for ESD of depressed-type gastric cancer between August 2011 and July 2014. Inclusion criteria were: (1) age 20 years or older; and (2) pathologically diagnosed gastric cancer that was eligible for ESD. The indication for ESD was based on the Japanese guidelines for ESD and endoscopic mucosal resection for early gastric cancer [2]. Exclusion criteria were: (1) exposure to chemotherapy or radiation therapy; (2) lack of tolerance to prolonged sedation; (3) lesions challenging to treat with ESD, such as ulcerative findings, size and location; (4) poor-quality endoscopic images; (5) macroscopic type protruding or flat type; and (6) difficult-to-diagnose histological findings. All eligible patients were recruited at the participating institutions, and all provided written informed consent.

\section{Endoscopy system and setting}

The instruments used in the current study were a magnification videoendoscopic system (GIF-H260Z, Olympus Medical Systems Co., Ltd., Tokyo, Japan) and standard optical videoendoscopic system (Evis Lucera Spectrum system; Olympus Medical Systems). Structural enhancement by the endoscopic video processor was set to A-mode level 8 for NBI-ME. The color mode was fixed at level 1 . To obtain stable endoscopic images at maximal magnification, a tip hood (Elastic Touch M, Top Corp., Tokyo, Japan) was mounted at the tip of the endoscope prior to the examination.

\section{Endoscopic diagnosis}

All endoscopic diagnoses were made by expert endoscopists who had diagnosed gastric cancer in more than 100 cases using magnification, just before ESD. A 3-mm area at the oral edge of the lesion was selected as the target area to diagnose histological type. The target area was limited to the oral edge of the lesion to reduce selection bias. Histological type of the target area was then diagnosed using non-magnified white light endoscopy (WLE). Two marks were placed between the target area to compare endoscopic and histological findings. Histological type of the target area was subsequently diagnosed by $\mathrm{NBI}$-ME under full water immersion. In the final step, $3 \%$ acetic acid was sprayed on the lesion through the forceps channel of the endoscope and the histological type of the target area was diagnosed by NBI-ME under acetic acid-enhanced conditions (NBI-AA). The process of endoscopic diagnosis is shown in - Fig. 1. Histological types were classified into differentiated adenocarcinoma and undifferentiated adenocarcinoma. Papillary adenocarcinoma and tubular adenocarcinoma were treated as differentiated adenocarcinoma. Poorly differentiated adenocarcinoma and signet-ring cell adenocarcinoma were treated as undifferentiated adenocarcinoma. Mucinous adenocarcinoma was excluded in the present study. 


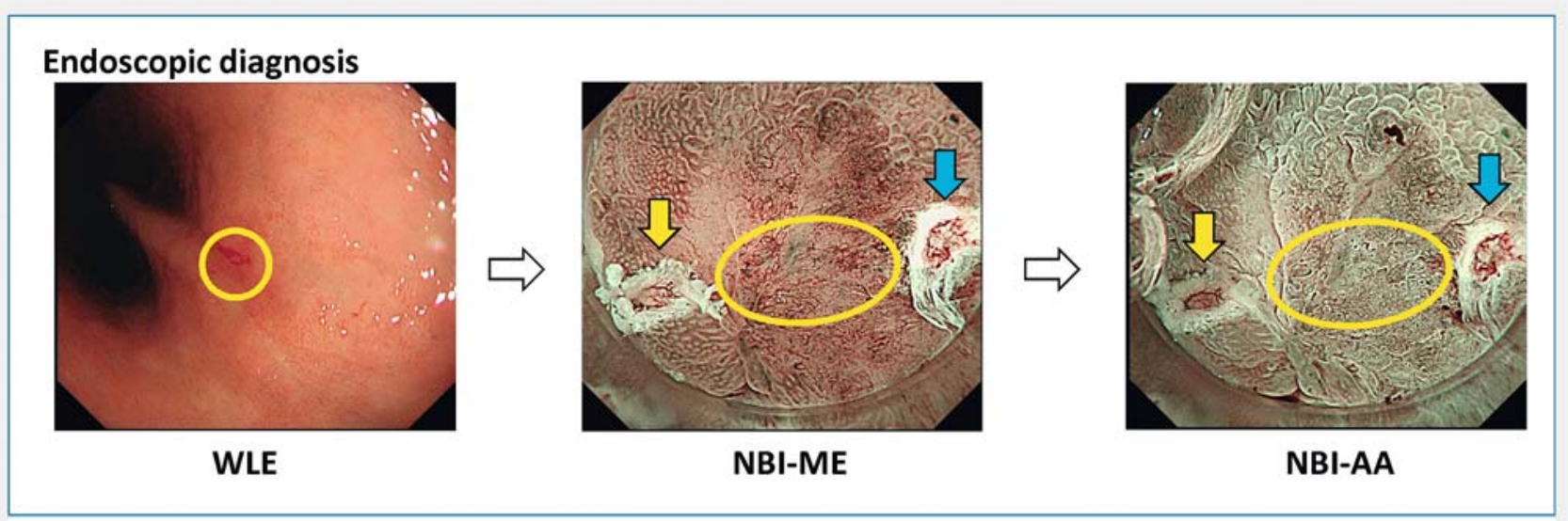

\section{Pathological diagnosis}
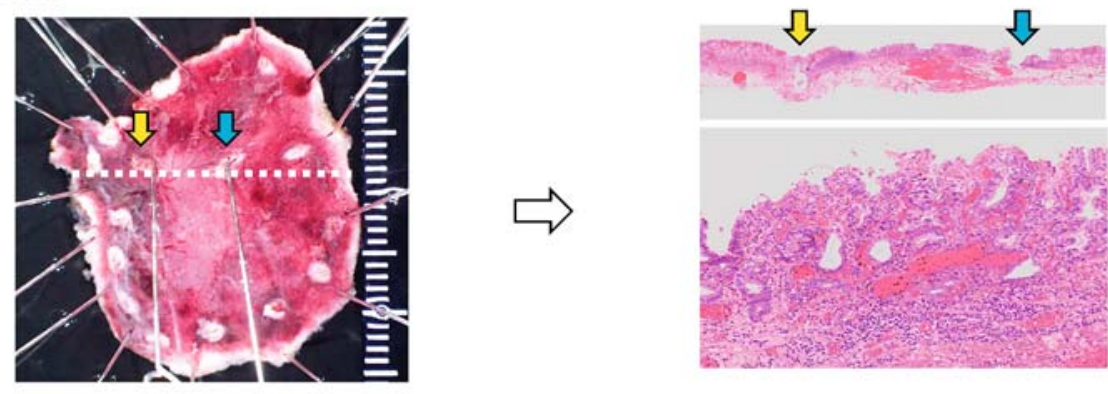

- Fig. 1 Flowchart of endoscopic and pathological diagnoses. Endoscopic diagnosis: A 3-mm area in the oral part of the lesion was selected as the target area to diagnose histological type. Two marks (target marks) were placed between the target area. Histological type of the target area was diagnosed by WLE, followed by NBI-ME and NBI-AA. Pathological diagnosis: The specimen was cut beside the target marks to compare endoscopic and histological findings. An expert pathologist diagnosed histological type of the target area without information on the endoscopic diagnosis.

\section{Diagnostic algorithm by WLE}

In WLE, demarcated red or whitish lesions and undemarcated whitish lesions were diagnosed as differentiated adenocarcinoma and undifferentiated adenocarcinoma, respectively ( Fig. 2).

\section{Diagnostic algorithm by NBI-ME}

NBI-ME findings were classified according to vascular and surface patterns [17]. Vascular pattern was diagnosed based on vascular tortuousness, caliber variability, and presence or absence of vascular networks ( $\$$ Fig. $\mathbf{3}$ ). If vascular networks were present, the lesion was diagnosed as differentiated adenocarcinoma. If vascular networks were absent, histology was diagnosed based on vascular irregularities. Vascular irregularities were classified based on severity into mild and severe ( $\triangleright$ Fig.3). If lesions showed microvessels running simply with homogeneous diameters, vascular irregularity was defined as mild. If microvessels branched in a complex manner, running tortuously, the so-called "corkscrew pattern" [13], vascular irregularity was defined as severe.

Surface patterns were grouped into villi, pit, and unclear patterns [17]. Villi pattern was defined as a round or oval struc- ture surrounding a white zone, while the pit pattern was defined as a white round structure by NBI-ME ( $\triangleright$ Fig. 3 ). When the villi pattern was identified, the lesion was diagnosed as differentiated adenocarcinoma. When the pit pattern was identified, the histological type was classified as either differentiated adenocarcinoma or undifferentiated adenocarcinoma based on the surface structure. When a regularly shaped pit pattern was recognized, the lesion was diagnosed as undifferentiated adenocarcinoma because the surface was covered by a non-neoplastic foveolar epithelium when the tumor volume was small [18]. The diagnostic algorithm by NBI-ME is shown in > Fig. 4 .

\section{Diagnostic algorithm by NBI-AA}

In NBI-AA, histology was diagnosed based on surface patterns [17]. When the surface pattern was unclear, the lesion was diagnosed as undifferentiated adenocarcinoma. The diagnostic algorithm by NBI-AA is shown in $>$ Fig. $\mathbf{5}$. 


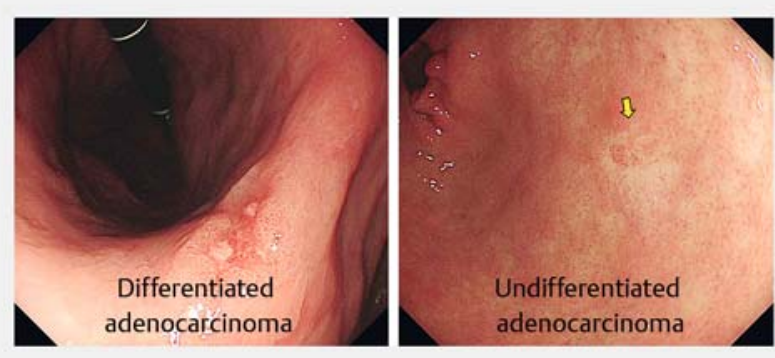

Fig. 2 Diagnostic algorithm by white light endoscopy. Demarcated red or whitish lesions and undemarcated whitish lesions were diagnosed as differentiated adenocarcinoma and undifferentiated adenocarcinoma, respectively. The yellow arrow indicates an undemarcated whitish lesion.

\section{Resection of lesions and treatment of resected specimens}

After marking and reaching a diagnosis, the lesion was resected by ESD. Procedures for and devices used in ESD were selected by the operators. The resected specimen was pinned on a rubber plate and fixed by formalin. Photos of the specimen were taken by stereoscopy. After fixation by formalin, the specimen was cut beside the target marks to compare endoscopic and histological findings.

\section{Pathological diagnosis}

The process of reaching a pathological diagnosis is shown in - Fig. 1. An expert pathologist (S.T.) diagnosed histological type of the target area based on hematoxylin eosin staining without information on the endoscopic diagnosis. In cases of mixed histological type, the main histological type was adopted.

\section{Diagnostic accuracy}

Diagnostic accuracy was defined as percentage concordance between the histological type and endoscopic diagnosis. Diagnostic sensitivity, specificity, positive predictive value (PPV), and negative predictive value (NPV) for each histological type were defined as the rate of correct diagnoses for each histological type, the rate of other histological types being correctly diagnosed, the rate of endoscopic diagnoses correctly corresponding to each histological type, and the rate of endoscopic diagnoses correctly corresponding to the other histological types, respectively.

\section{Subanalysis}

We evaluated PPV for each endoscopic finding in NBI-ME and NBI-AA. In NBI-ME, we assessed PPV for villi pattern, irregular pit pattern, regular pit pattern, unclear surface pattern with a vascular network, unclear surface pattern with mild vascular irregularity, and unclear surface pattern with severe vascular irregularity. In NBI-AA, we evaluated PPV for villi pattern, irregular pit pattern, regular pit pattern, and unclear surface pattern.

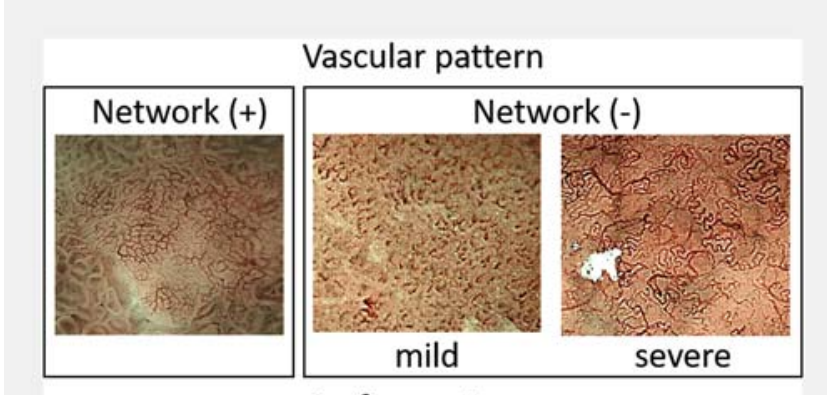

Surface pattern

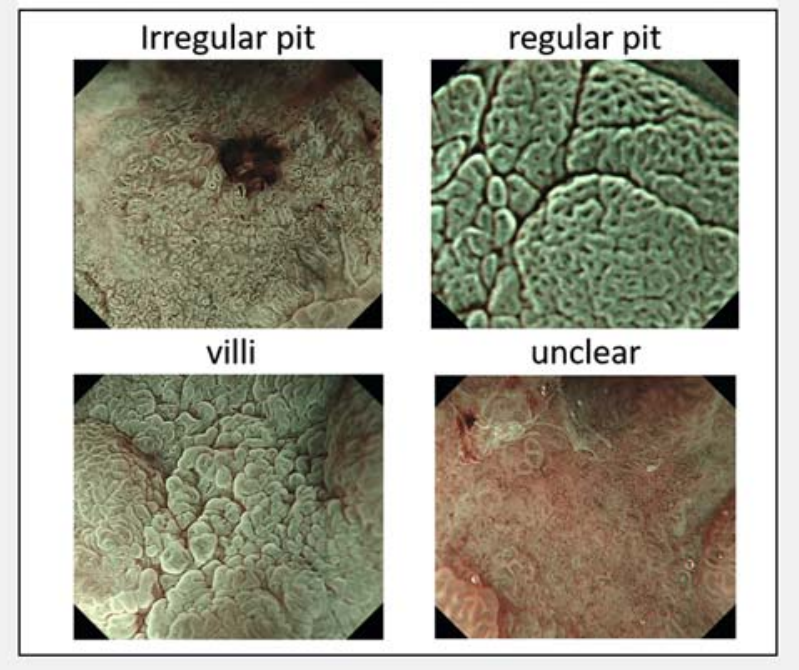

- Fig.3 Vascular and surface patterns. The vascular pattern was diagnosed based on vascular tortuousness, caliber variability, and the presence or absence of vascular networks. If vascular networks were present, the lesion was diagnosed as differentiated adenocarcinoma. If vascular networks were absent, histology was diagnosed based on vascular irregularities. Vascular irregularities were classified based on severity into mild and severe. If lesions showed microvessels running simply with homogeneous diameters, vascular irregularity was defined as mild. If microvessels branched in a complex manner, running tortuously, the so-called "corkscrew pattern," vascular irregularity was defined as severe. Surface patterns were grouped into villi, pit, and unclear patterns. Villi pattern was defined as a round or oval structure surrounding a white zone, while the pit pattern was defined as a white round structure by NBI-ME.

\section{Sample size calculation}

We performed a retrospective study to compare diagnostic accuracy of NBI-ME and NBI-AA for histological type in early gastric cancer [19]. Based on the results of this study, we estimated the diagnostic accuracy of WLE, NBI-ME, and NBI-AA for the histological type to be $55 \%, 60 \%$, and $80 \%$, respectively. To confirm the superiority of NBI-AA to WLE and NBI-ME for diagnostic accuracy with a clinically meaningful difference of $10 \%$ in diagnostic accuracy, we calculated that a sample size of 226 cases was needed with a statistical power of $80 \%$ and significance level of alpha of $5 \%$. Therefore, 250 patients were enrolled to account for dropouts. 


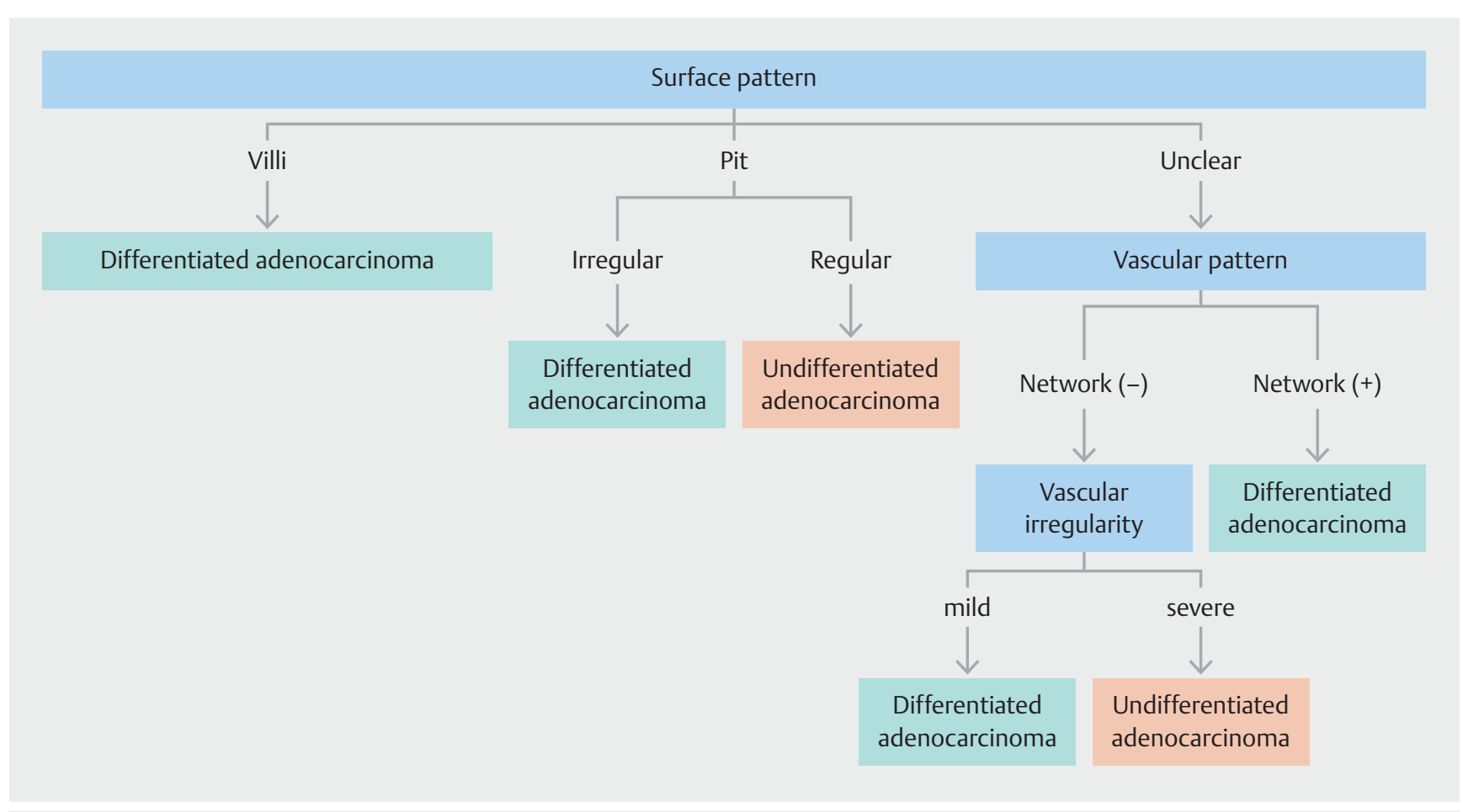

Fig. 4 Diagnostic algorithm by NBI-ME.

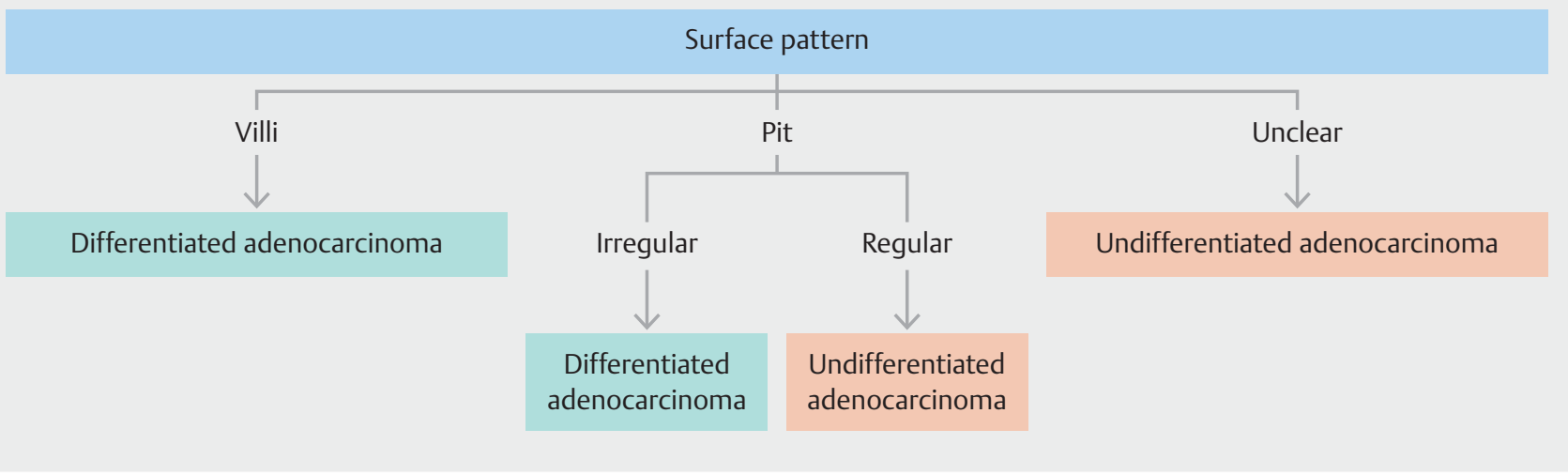

- Fig. 5 Diagnostic algorithm by NBI-AA.

\section{Statistical analysis}

The diagnostic accuracy of each modality was statistically analyzed with an adequate sample size using the chi-squared test. The significance level was set to $P<0.05$. The diagnostic accuracy of each modality was expressed as point estimates of the accuracy rate with a $95 \%$ confidence interval $(\mathrm{Cl})$. All statistical analyses were performed with EZR (Saitama Medical Center, Jichi Medical University, Saitama, Japan), which is a graphical user interface for $R$ (The R Foundation for Statistical Computing, Vienna, Austria). More precisely, it is a modified version of $\mathrm{R}$ commander designed to add statistical functions frequently used in biostatistics.

\section{Results}

A total of 250 cases were enrolled in the current study. Histological findings were difficult to diagnose in 18 cases. Endoscopic images of six cases were of poor quality because of the adherence of mucus and blood clots. ESD was canceled in five cases. These 29 cases were excluded from the current study. Therefore, 221 cases were analyzed ( $\triangleright$ Fig. 6 ). Histological types of the target areas were differentiated adenocarcinoma and undifferentiated adenocarcinoma in 206 and 15 cases, respectively. Clinicopathologic features of these lesions are summarized in $>$ Table 1. 


\section{0 depressed-type gastric cancers enrolled \\ Excluded: 29 cases \\ $\rightarrow$. Unsatisfied analysis: 24 cases \\ - Cancellation of ESD: 5 cases \\ 221 cases analyzed}

- Fig. 6 Flowchart of enrollment and analysis.

\section{Diagnostic accuracy}

Endoscopic and pathological diagnoses by and diagnostic accuracy of each modality were shown in $>$ Table 2 . Diagnostic accuracy of histological type for WLE, NBI-ME, and NBI-AA was 96.4\% (213/221), 96.8\% (214/221), and 95.5\% (211/221), respectively. No significant differences were observed between the modalities. ( $P=1$ for WLE vs NBI-ME, $P=0.622$ for NBI-ME vs NBI-AA, $P=0.811$ for NBI-AA vs WLE)

\section{Diagnostic sensitivity, specificity, PPV, and NPV by each modality}

According to the histological type, the diagnostic sensitivity, specificity, PPV, and NPV of each modality are shown in $>$ Table 3. Diagnostic sensitivity, specificity, PPV, and NPV $(95 \% \mathrm{Cl})$ of WLE for differentiated adenocarcinoma were $99.0 \%$ (96.5-
- Table 1 Clinicopathologic features of 221 early gastric cancer lesions.

\begin{tabular}{|l|c|c|}
\hline & $\begin{array}{l}\text { Differentiated } \\
\text { adenocarcinoma } \\
\text { (n= 206) }\end{array}$ & $\begin{array}{l}\text { Undifferentiated } \\
\text { adenocarcinoma } \\
\text { (n=15) }\end{array}$ \\
\hline Macroscopic type & & \\
\hline - Depressed & 206 & 15 \\
\hline - Flat & 0 & 0 \\
\hline - Protruding & 0 & 0 \\
\hline Size, median (range), mm & $12(3-70)$ & $15(6-30)$ \\
\hline Location & $22(10.7)$ & $2(13.3)$ \\
\hline - Upper third, \% & $68(33.0)$ & $8(53.4)$ \\
\hline - Middle third, \% & $116(56.3)$ & $5(33.3)$ \\
\hline - Lower third, \% & & \\
\hline Invasive depth & $182(88.3)$ & $11(73.3)$ \\
\hline - Mucosa, \% & $24(11.7)$ & $4(26.7)$ \\
\hline - Submucosa, \% & & \\
\hline
\end{tabular}

99.9), $60.0 \%$ (32.3-83.7), $97.1 \%$ (93.9-98.9), and $81.8 \%$ (48.2-97.7), respectively. Diagnostic sensitivity, specificity, PPV, and NPV $(95 \% \mathrm{Cl})$ of NBI-ME for differentiated adenocarcinoma were $99.5 \%(97.3-100), 60.0 \%(32.3-83.7), 97.2 \%$

\begin{tabular}{|c|c|c|c|}
\hline & & \multicolumn{2}{|l|}{ Endoscopic diagnosis } \\
\hline WLE & & Differentiated adenocarcinoma & Undifferentiated adenocarcinoma \\
\hline \multirow{2}{*}{ Pathological diagnosis } & Differentiated adenocarcinoma & 204 & 2 \\
\hline & Undifferentiated adenocarcinoma & 6 & 9 \\
\hline Diagnostic accuracy (95\% Cl) & \multicolumn{3}{|l|}{$96.4 \%(213 / 221)(93.0-98.4)$} \\
\hline \multicolumn{4}{|l|}{ NBI-ME } \\
\hline & & \multicolumn{2}{|l|}{ Endoscopic diagnosis } \\
\hline & & Differentiated adenocarcinoma & Undifferentiated adenocarcinoma \\
\hline \multirow{2}{*}{ Pathological diagnosis } & Differentiated adenocarcinoma & 205 & 1 \\
\hline & Undifferentiated adenocarcinoma & 6 & 9 \\
\hline Diagnostic accuracy (95\% Cl) & \multicolumn{3}{|l|}{$96.8 \%(214 / 221)(93.6-98.7)$} \\
\hline \multicolumn{4}{|l|}{ NBI-AA } \\
\hline & & \multicolumn{2}{|l|}{ Endoscopic diagnosis } \\
\hline & & Differentiated adenocarcinoma & Undifferentiated adenocarcinoma \\
\hline \multirow{2}{*}{ Pathological diagnosis } & Differentiated adenocarcinoma & 202 & 4 \\
\hline & Undifferentiated adenocarcinoma & 6 & 9 \\
\hline Diagnostic accuracy (95\% Cl) & $95.5 \%(211 / 221)(91.8-97.8)$ & & \\
\hline
\end{tabular}


- Table 3 Diagnostic sensitivity, specificity, PPV, and NPV for the differentiated type.

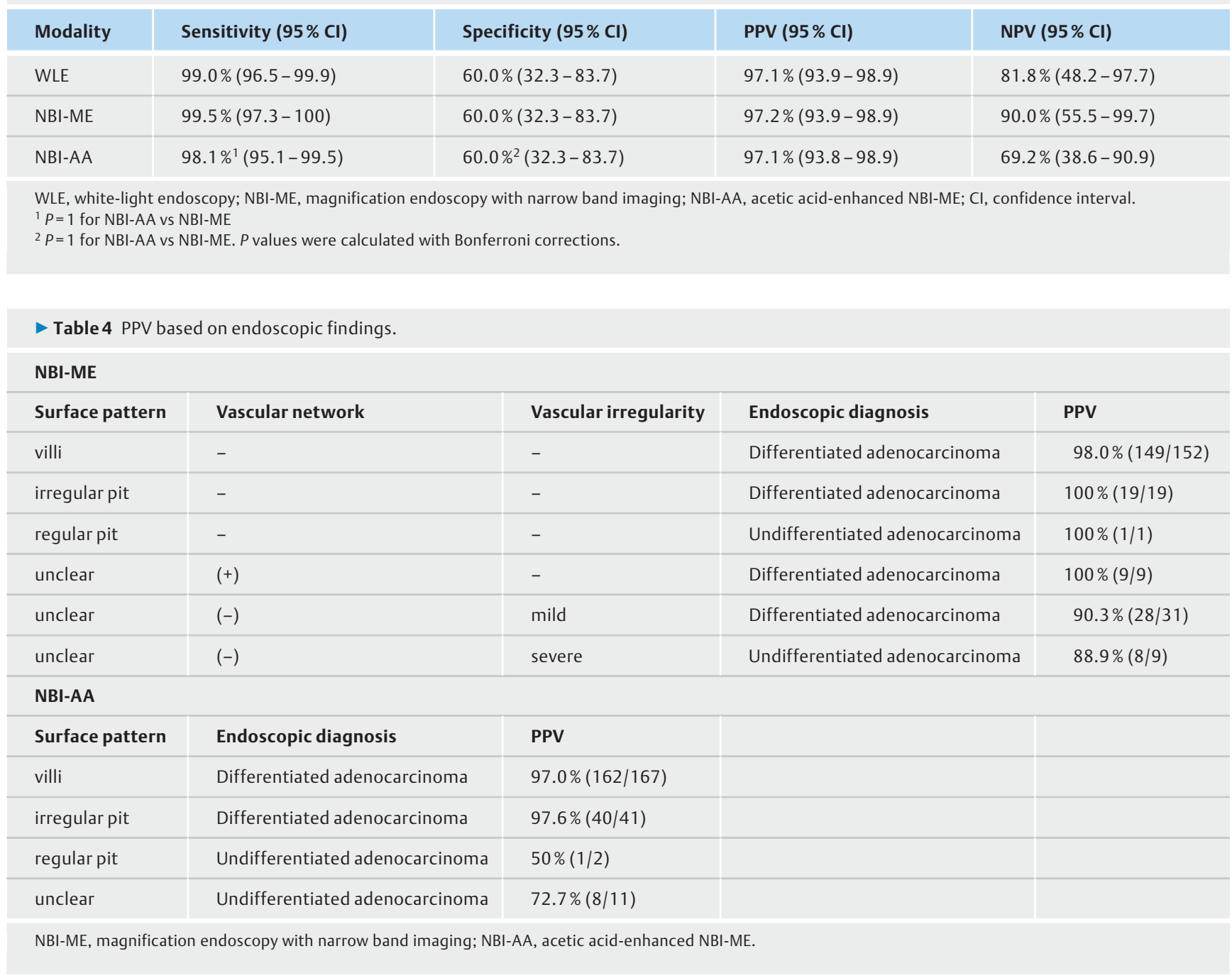

(93.9-98.9), and 90.0\% (55.5-99.7), respectively. Diagnostic sensitivity, specificity, PPV, and NPV $(95 \% \mathrm{Cl})$ of NBI-AA for differentiated adenocarcinoma were $98.1 \%$ (95.1-99.5), $60.0 \%$ (32.3-83.7), 97.1\% (93.8-98.9), and 69.2\% (38.6-90.9), respectively. No significant differences were observed between the modalities (sensitivity $P=1$ for NBI-AA vs NBI-ME; specificity $P=1$ for NBI-AA vs NBI-ME. $P$ values were calculated with Bonferroni corrections).

\section{Subanalysis}

PPV based on endoscopic findings in NBI-ME was 98.0\% (149/ $152)$ for the villi pattern, $100 \%$ (19/19) for the irregular pit pattern, $100 \%$ (1/1) for the regular pit pattern, $100 \%$ (9/9) for the unclear surface pattern with a vascular network, $90.3 \%$ (28/31) for the unclear surface pattern with mild vascular irregularity, and $88.9 \%$ (8/9) for the unclear surface pattern with severe vascular irregularity. PPV based on endoscopic findings in NBI-AA were $97.0 \%$ (162/167) for the villi pattern, $97.6 \%$ (40/41) for the irregular pit pattern, $50 \%$ (1/2) for the regular pit pattern, and $72.7 \%(8 / 11)$ for the unclear surface pattern ( $\triangleright$ Table 4$)$.

\section{Discussion}

The usefulness of NBI-ME for histologically diagnosing gastric cancer has already been demonstrated [12-14]. However, a multicenter prospective study has not yet been conducted on the diagnostic accuracy of the histological type by endoscopy. Therefore, we herein performed a multicenter prospective study to evaluate the usefulness of NBI-ME and NBI-AA for diagnosing histological type of depressed-type early gastric cancer. Most protruded-or polypoid-type early gastric cancers are histologically classified as well-differentiated tubular adenocarcinomas. Poorly differentiated adenocarcinomas are unusual among macroscopically protruded-type early gastric cancers [20]. Therefore, we focused on depressed-type early gastric cancers and excluded protruded-type early gastric cancers in the present study.

The key feature of the current study is that we placed two marks between the target area to strictly compare endoscopic and histological findings. This is very challenging to achieve in surgical cases because patients need to undergo endoscopy for marking the day before surgery. However, this places a burden 
on patients. Therefore, surgical cases were not included in the current study. To date, there has been no multicenter prospective study similar to the current study. Therefore, this study is unique.

No significant difference was observed in diagnostic accuracy of the histological type among WLE, NBI-ME, and NBI-AA in the current study. The most plausible reason for this was that the diagnostic accuracy of WLE was higher (96.4\%) than expected. High diagnostic accuracy was achieved because: 1) the histological type of the target area was mainly differentiated adenocarcinoma (93.2\%: 206/221); and 2) sensitivity for differentiated adenocarcinoma by WLE was very high (99.0\%).

Based on the findings of a retrospective study [19], we estimated that the diagnostic accuracy of WLE, NBI-ME, and NBIAA for the histological type was $55 \%, 60 \%$, and $80 \%$, respectively. However, the diagnostic accuracy of WLE, NBI-ME, and NBI-AA for the histological type was $96.4 \%, 96.8 \%$, and $95.5 \%$, respectively, in the current study. These two studies suggested that the retrospective study did not reflect the diagnostic accuracies obtained in the current study.

On the other hand, specificities for differentiated adenocarcinoma, that is sensitivities for undifferentiated adenocarcinoma, by each modality were lower (WLE 60.0\%, NBI-ME 60.0\%, and $\mathrm{NBI}-\mathrm{AA} 60.0 \%$ ) than those sensitivities for differentiated adenocarcinoma. The results of the current study demonstrated that it was difficult to diagnose undifferentiated adenocarcinoma, even by NBI-ME and NBI-AA. Therefore, we considered biopsies to still be necessary for diagnosing histological type and informing decision-making regarding treatment strategies (endoscopic resection or surgery) for gastric cancer.

In the current study, there were 11 misdiagnosed cases in NBI-ME and NBI-AA (NBI-ME and NBI-AA: 6 cases, NBI-AA: 4 cases, NBI-ME: 1 case). The main causes for these misdiagnoses were as follows: 1) difficulties associated with endoscopic observations due to mucus and blood (5 cases); 2 ) misdiagnosis of endoscopic findings (4 cases); and 3) discrepancies in histological findings between the surface and deep layers (2 cases). Difficulties associated with endoscopic observations due to mucus and blood were identified as the main cause. We considered even a small amount of mucus and blood to increase difficulty associated with diagnosing the histological type.

When the villi pattern, irregular pit pattern, and vascular network were observed by NBI-ME or NBI-AA, histological type was mostly differentiated adenocarcinoma in the current study (PPV was $97 \%$ or higher). This result suggested that the villi pattern, irregular pit pattern, and vascular network may be useful for identifying differentiated adenocarcinoma. On the other hand, PPV was lower for the unclear surface pattern without a vascular network. When the surface pattern was unclear, it was difficult to diagnose histological type because histological type of gastric cancer is diagnosed by degree of structural atypia [21]. Yagi et al. reported that the microsurface pattern was not visualized by NBI-ME when the intervening parts were short or the crypts were shallow [22]. In these cases, NBI-AA enables clear observations of the microsurface pattern and evaluations of structural atypia. While 49 cases showed the unclear surface pattern by NBI-ME in the current study, surface patterns be- came detectable in 38 of 49 cases with NBI-AA. These results suggested that NBI-AA is more useful for structural evaluations than NBI-ME. However, NBI-AA did not improve PPV of these 49 cases (NBI-ME 91.8\%: 45/49, NBI-AA 85.7\%: 42/49). These results suggest that we need to evaluate not only the surface pattern, but also the vascular pattern for histological diagnosis when NBI-ME shows the unclear surface pattern.

Shibagaki et al. performed a prospective cross-sectional study to compare diagnostic accuracy of WLE, NBI-ME, and $\mathrm{NBI}-\mathrm{AA}$ for specific histological types in gastric mucosal neoplasms [23]. In that study, diagnostic accuracy of NBI-AA was significantly higher than those of the other modalities. They suggested that NBI-AA needs to be performed when the surface pattern is unclear by NBI-ME. However, the current study did not prove this hypothesis. We intend to perform a prospective study to compare diagnostic accuracy of WLE, NBI-ME, and $\mathrm{NBI}-\mathrm{AA}$ for specific histological types in early gastric cancer with the unclear surface pattern using NBI-ME.

In the current study, histological type was mainly differentiated adenocarcinoma and the proportion of undifferentiated adenocarcinoma cases was very small because this study only included ESD candidates. We considered the inclusion criteria to have resulted in a large selection bias, which was a significant limitation of the current study. The other limitations are as follows: 1) unblinded pathological diagnosis of biopsy samples; 2) unblinded result of diagnoses by WLE in subsequent evaluations by NBI-ME and NBI-AA; and 3) endoscopists who were experts.

\section{Conclusion}

In conclusion, NBI-ME and NBI-AA did not show any advantages over WLE for overall diagnostic accuracy in the current study. Villi pattern, irregular pit pattern, and vascular network may be useful for identifying differentiated adenocarcinoma.

\section{Acknowledgements}

We are grateful to Tadakazu Shimoda, PCL Japan for the pathological diagnosis in this study. This study was supported by the Japanese Foundation for Research and Promotion of Endoscopy.

\section{Competing interests}

None

References

[1] Gotoda T, Jung HY. Endoscopic resection (endoscopic mucosal resection/endoscopic submucosal dissection) for early gastric cancer. Dig Endosc 2013; 25: (Suppl. 01): 55-63

[2] Ono H, Yao K, Fujishiro M et al. Guidelines for endoscopic submucosal dissection and endoscopic mucosal resection for early gastric cancer. Dig Endosc 2016; 28: 3 - 15

[3] Gotoda T. Endoscopic resection of early gastric cancer. Gastric Cancer 2007; 10: $1-11$ 
[4] Hirasawa T, Gotoda T, Miyata S et al. Incidence of lymph node metastasis and the feasibility of endoscopic resection for undifferentiatedtype early gastric cancer. Gastric Cancer 2009; 12: 148-152

[5] Martin IG, Dixon MF, Sue-Ling $\mathrm{H}$ et al. Goseki histological grading of gastric cancer is an important predictor of outcome. Gut 1994; 35 : $758-763$

[6] Allum WH, Blazeby JM, Griffin SM et al. Guidelines for the management of oesophageal and gastric cancer. Gut 2011; 60: 1449-1472

[7] Lee JH, Kim JH, Rhee K et al. Undifferentiated early gastric cancer diagnosed as differentiated histology based on forceps biopsy. Pathol Res Pract 2013; 209: $314-318$

[8] Kaise M, Kato M, Urashima M et al. Magnifying endoscopy combined with narrow-band imaging for differential diagnosis of superficial depressed gastric lesions. Endoscopy 2009; 41: 310 - 315

[9] Yao K, Anagnostopoulos GK, Ragunath K. Magnifying endoscopy for diagnosing and delineating early gastric cancer. Endoscopy 2009; 41 : $462-467$

[10] Ezoe $\mathrm{Y}$, Muto M, Uedo N et al. Magnifying narrowband imaging is more accurate than conventional white-light imaging in diagnosis of gastric mucosal cancer. Gastroenterology 2011; 141: 2017 - 2025 e3

[11] Nagahama T, Yao K, Maki S et al. Usefulness of magnifying endoscopy with narrow-band imaging for determining the horizontal extent of early gastric cancer when there is an unclear margin by chromoendoscopy (with video). Gastrointest. Endosc 2011; 74: 1259-1267

[12] Nonaka K, Arai S, Ban S et al. Prospective study of the evaluation of the usefulness of tumor typing by narrow band imaging for the differential diagnosis of gastric adenoma and well-differentiated adenocarcinoma. Dig. Endosc 2011; 23: $146-152$

[13] Nakayoshi T, Tajiri H, Matsuda K et al. Magnifying endoscopy combined with narrow band imaging system for early gastric cancer: correlation of vascular pattern with histopathology (including video). Endoscopy 2004; 36: $1080-1084$
[14] Yokoyama A, Inoue H, Minami H et al. Novel narrow-band imaging magnifying endoscopic classification for early gastric cancer. Dig Liver Dis 2010; 42: $704-708$

[15] Kadowaki S, Tanaka K, Toyoda H et al. Ease of early gastric cancer demarcation recognition: a comparison of four magnifying endoscopy methods. J. Gastroenterol. Hepatol 2009; 24: 1625-1630

[16] Yagi K, Aruga Y, Nakamura A et al. The study of dynamic chemical magnifying endoscopy in gastric neoplasia. Gastrointest Endosc 2005; 62: $963-969$

[17] Oyama T (editor) Endoscopic diagnosis of superficial gastric cancer for ESD. Springer; 2016

[18] Okada K, Fujisaki J, Kasuga A et al. Diagnosis of undifferentiated type early gastric cancers by magnification endoscopy with narrow-band imaging. J. Gastroenterol Hepatol 2011; 26: 1262 - 1269

[19] Oyama T, Tomori A, Kishino T et al. Histopathological Diagnosis of Gastric Cancers by Magnifying Endoscopy with NBI. Stomach and Intestine 2011; 46: 933 - 942 (Japanese-language article)

[20] Sakane M, Kashiwagi R, Mitsutsuji M et al. A case of poorly differentiated early adenocarcinoma of the stomach forming a protruded type lesion. Digestive Endoscopy 1997; 9: 300 - 304

[21] Japanese classification of gastric carcinoma: 3rd English edition. Gastric Cancer 2011; 14: 101 - 112

[22] Yagi K, Nozawa Y, Endou S et al. Diagnosis of early gastric cancer by magnifying endoscopy with NBI from viewpoint of histological imaging: mucosal patterning in terms of white zone visibility and its relationship to histology. Diagn Ther Endosc 2012; 2012: 954809

[23] Shibagaki K, Amano Y, Ishimura N et al. Diagnostic accuracy of magnification endoscopy with acetic acid enhancement and narrow-band imaging in gastric mucosal neoplasms. Endoscopy 2016; 48: 16-25 